\title{
Effects of Tailored Resistance Exercise Training in a Group of Metalworkers with Ergonomic or Manual Handlings Loads Prescription by the Occupational Physician: a Pilot Study
}

\author{
S. Gobbo ${ }^{*}$, V. Bullo ${ }^{1 *}$, E. Roma ${ }^{1}$, M. Bergamo ${ }^{1}$, B. Vendramin ${ }^{1}$, F. Duregon ${ }^{1}$, L. Cugusi ${ }^{2}$ \\ A. Di Blasio 3 , S. Maso 4 , D. Sales Bocalini ${ }^{5}$, C. L. Alberton ${ }^{6}$, G. Priolo, D. Cruz-Diaz ${ }^{8}$, A. \\ Ermolao1, M. Bergamin* \\ * these authors equally contributed to the manuscript. \\ 1 Sport and Exercise Medicine Division, Department of Medicine, University of Padova, Padova, Italy \\ 2 Department of Biomedical Sciences, University of Sassari, Sassari, Italy \\ ${ }^{3}$ Department of Medicine and Sciences of Aging, G. d'Annunzio, University of Chieti-Pescara, Pescara, Italy \\ ${ }^{4}$ Department of Cardiac, Thoracic and Vascular Sciences, University of Padova, Padova, Italy \\ ${ }^{5}$ Laboratorio de Fisiologia e Bioquimica Experimental, Centro de Educacao Fisica e Deportos, Universidade \\ Federal do Espirito Santo (UFES), Vitoria, Espirito Santo, Brazil \\ 6 Physical Education School, Federal University of Pelotas, Rio Grande do Sul, Brazil \\ 7 Freelance professional \\ 8 Department of Health Sciences, Faculty of Health Sciences, University of Jaén, Jaén, Spain
}

\section{CORRESPONDING AUTHOR:}

Marco Bergamin

Sport and Exercise Medicine Division

Department of Medicine

University of Padova

via Giustiniani 2

35128 Padova, Italy

E-mail: marco.bergamin@unipd.it

\section{DOI:}

10.32098/mltj.01.2021.19

LEVEL OF EVIDENCE: 4

\begin{abstract}
SUMMARY
Background. Work-related musculoskeletal disorders (WRMSDs) are cause of disability and loss of productivity. Part of the workers suffers from WRMSDs while others are exposed to risk factors. Resistance training (RT) can reduce pain symptomatology, counteracting WRMSDs. The aim of this investigation is to evaluate the effectiveness of a RT program in a group of metalworkers on pain perception, disability and physical fitness in context of real work-setting.

Methods. Eighteen metalworkers took part to a 12 weeks RT program with a personalized intervention during the sessions. Disability of the arm, shoulder and hand questionnaire (DASH) and the visual analogue scale for the low back (L-VAS) represent the primary outcome regarded. Handgrip strength, shoulder and hamstrings flexibility and cardiorespiratory endurance were tested. Pre-post differences were evaluated through T-test.

Results. DASH score improved significantly of 4.28 points $(\mathrm{p}=0.03)$. L-VAS did not change ( $\mathrm{p}=0.38)$ while physical fitness outcomes improved significantly except for the shoulder flexibility. Spontaneous attendance reached $83.42 \%$.

Conclusions. Pain and disability decreased in upper limb and shoulder but not in the lumbar spine. Moreover, physical fitness of participants improved. Considering the spontaneous attendance to the program of these participants, we exhort RT implementation in occupational primary and secondary prevention programs.
\end{abstract}

\section{KEY WORDS}

Musculoskeletal disorders; low back pain; exercise; workplace. 


\section{INTRODUCTION}

Work-related musculoskeletal disorders (WRMSDs) can be defined as abnormalities in the soft tissue of upper and lower limbs bones and joints, such as spine (1). Several pathomechanisms act at the organ and tissue levels, and provoke WRMSDs. The repetitive movement during the workday could induce a muscular imbalance due to the overuse of specific muscular group and the underuse of other (2). In addition, the maintenance of prolonged and/or abnormal static position could affect peripheral nerves increasing pressure or tension causing chronic compression. Without muscle restoration, this "bad conduct" could turn on a circle of swelling, inflammation and micro-circle impairment that induce a nerve dysfunction (2).

Incidence and prevalence of WRMSDs have been increasing through the years. Between 2006 and 2010 in Italy the notifications of WRMSDs augmented by $158 \%$ (3), whit an increase of work-related disability and loss of productivity. Furthermore, they could affect activities of daily living (ADL) and quality of life (QoL) (4). Actions of prevention are therefore needed to lower the incidence of WRMSDs, and for that present pain, to reduce symptomatology.

Increase physical activity has been showed to be beneficial for several musculoskeletal diseases, with particular efficacy for the reduction of low back pain (LBP) (5); on the contrary, for upper limb and neck musculoskeletal disorders research reports contrasting results. In details, there is evidence consensus about the beneficial effects of physical exercise training (6), especially resistance training interventions $(3,6)$. The effect of resistance training on pain relief seems to be attributable to acceleration of protein synthesis and degradation, leading to reconstruction of abnormal or painful muscle tissue (7) or to reduction in relative workload due to strength improvement (8). Moreover, the level of physical activity is inversely associated with LBP (9).

The aim of this pilot study is to evaluate the effectiveness of a supervised resistance training program on individual pain perception, disability and physical fitness in a group of metalworkers reporting pain symptoms. A second point of interest is to verify the feasibility of the intervention performed into the company considering individual conditions, the strenuous working activity and the full freedom of choice to take part to the training sessions.

\section{MATERIALS AND METHODS}

\section{Participants}

This pilot study was performed between October and December 2017. Twenty-two male metalworkers were recruited from an Italian steel company to participate in 12 weeks of adapted physical exercise (PE) protocol. Inclusion criteria were: 1 ) age $\geq 18$ years old, 2) ergonomic or manual handlings of loads prescription by the occupational physician, 3) history of musculoskeletal pain, 4) no concurrent participation in other exercise program. Written informed consent was obtained prior to enrollment. The study complied with the current laws of Italy for research on human participants and was approved by the local review board.

\section{Health-related physical fitness assessment}

The participants were evaluated at the same day, 7 days before and 7 days after PE intervention. Cardiorespiratory endurance was assessed through 2-minutes step test (2MST). Subjects were asked to maintain upright stance near a wall and performed the greater number of full steps in place for two minutes, reaching with the knee a standardized height defined by the midpoint between patella and iliac crest. Each step requires the complete contact of the foot to the floor. Score is the number of times that right knee reaches the required height correctly (10).

Flexibility measures were taken to the nearest $1 \mathrm{~cm}$. Flexibility of the lower body (primary hamstring) was evaluated with Chair Sit and Reach Test (CSRT). The subjects had to set on the edge of the chair, with one leg flexed at $90^{\circ}$ and the other extended. They were asked to overlap the middle fingers of their hand with the palm facing down and to bend forward as far as they could, without bending the extended knee or changing ankle position. Score was measured in centimeters $(\mathrm{cm})$ and it was zero if the middle finger touches the tip of the foot, it has positive sign if the middle finger passes the tip of the foot or negative if it does not (10). The overall shoulder range of motion was evaluated with the Back-Scratch Test (BST). The subjects were asked to raise the arm, bent the elbow and reach across the back as far as possible. At the same time the other arm is placed down and behind the back. Subjects were asked to reach their fingers behind the back. An overlap is considered to produce a positive score, whereas a gap is considered a negative score. If middle fingers touch, the score is zero (10). Each flexibility test was performed three times for each side, right and left mean score were considered.

Muscular strength was tested with Handgrip Strength Test (HST). The HST is a direct measure of maximal isometric grip strength and it reflects overall body strength in untrained subjects (11). Subjects sit on a chair without armrest and elbow was flexed to $90^{\circ}$. Hydraulic dynamometer (Baseline, Elmsford, NY, USA) was used; grip was adjusted so that middle portion of the middle finger was placed in front of the handle. The fingers were in front of 
dynamometer handle, wrist and forearm laid in midprone position. Participants were asked to squeeze as hard as possible, for at least 5 seconds. Score was recorded at the nearest $1 \mathrm{~kg}$. Subjects undertook three trials for each hand, the mean score was considered. The rest period between trials were of three minutes to prevent any fatigue effect.

\section{Disability and symptom assessment}

Work-related musculoskeletal symptoms were evaluated with the Disability of the Arm, Shoulder and Hand Questionnaire (DASH) (12) and the Visual Analogue Scale (VAS). The Italian version of DASH is a 30-item disability/ symptom scale concerning the patient's health status during the preceding week. The items ask about the degree of difficulty in performing different physical activities due to arm, shoulder, or hand problems (21 items), the severity of each pain symptoms, activity-related pain, tingling, weakness and stiffness ( 5 items), as well as the impact on social activities, work, sleep, and self-image (4 items). The score of all items ranges from 1 to 5 and allows calculating a summary scale score ranging from 0 to 100 . Pain intensity of low back was evaluated with VAS. The VAS is a 0 to $10 \mathrm{~cm}$ line, where 0 corresponded to absence of pain, while 10 represents the worst imaginable pain (13). Subjects were asked to assess the level of pain they experienced in low back (L-VAS). A representation of the human body was used to clearly indicate body part to consider. All these questionnaires were self-administered. The occupational physician, after clinical interview and examination, identified the most painful area or joints, which showed higher-risk for pain.

\section{Physical activity assessment}

The level of physical activity and sedentary behavior were evaluated throughout the Global Physical Activity Questionnaire (GPAQ). It is a 16-items questionnaire composed by 3 domains: physical activity at work, physical activity for travel to and from places, and recreational physical activities (14).

\section{Exercise protocol}

Physical exercise protocol was performed for 12 weeks, supervised by an exercise specialist. The exercise session took place in the afternoon $(5.00 \mathrm{pm})$ at the end of the working schedule of participants. Each exercise session started with a 10-minutes of warm-up exercise focused on joint movement of mainly body districts (e.g., scapular elevation and depression, anterior and posterior pelvic tilt, etc.). Resistance exercises were performed for major muscu- lar groups, with elastic bands or free weights. To standardize the intensity, Borg's category ratio scale 10 (CR10) was used (15). CR10 ratings, referred to the perceived exertion of the prime movers, were measured at the end of the first set. If prescribed CR10 rate was reached in the first set, weight was maintained for the other sets, otherwise weight was adjusted, and another attempt was made. The session ended with a 10-minutes cool-down, in which stretching exercises for the main muscular groups were performed. Weeks 1-3 were employed for the exercises training. After this phase, the workout was adapted for each participant, including with specific exercises in regards to musculoskeletal disease and/or pain. In details, was added resistance and stretching exercises for neck, wrist, forearm, shoulders, and pelvic/hamstrings muscles. The aim was strengthening and increase flexibility to reduce pain. More information about training variables and progression are presented on table I.

\section{Statistical analysis}

Statistical analysis was performed with $\mathrm{R}$, version 3.5.2 (16). Results were expressed in mean and standard deviation as mean (SD), except for attendance that was expressed in mean and Interquartile range (IQR). A paired-sample Student's $\mathrm{T}$ Test was performed for each dependent variable. Significance limits were set at $\alpha=0.05$. Confidence intervals were reported. Effect size (ES) of each outcome was calculated according to the Cohen's $d$ formula: ES = (mean pre value - mean post value)/SD pre value. The ES is a measure of the effectiveness of a treatment and it helps to determine whether a statistically significant difference is a determined by the practical concern. Interpretation is based on Cohen (17) criteria where an ES value of 0.2 indicates a small effects, ES of 0.5 indicates moderate effect, and ES higher than 0.8 indicates a large effect. Attendance to the protocol will be expressed as the percent of sessions accomplished by the participants on the total number of sessions (24 lessons).

This study meet the ethical standards of Muscle, Ligaments and Tendons Journal (18).

\section{RESULTS}

Two participants dropped-out, and 18 metalworkers completed the PE intervention. Participant's baseline characteristics are presented into table II. All participants experienced LBP; moreover, 4 out 18 had upper limb disease, 5 out 18 had low back disease, and 1 had neck disease. According to GPAQ guidelines, 11 participants were active, 3 had low level of PA and 4 were sedentary. 


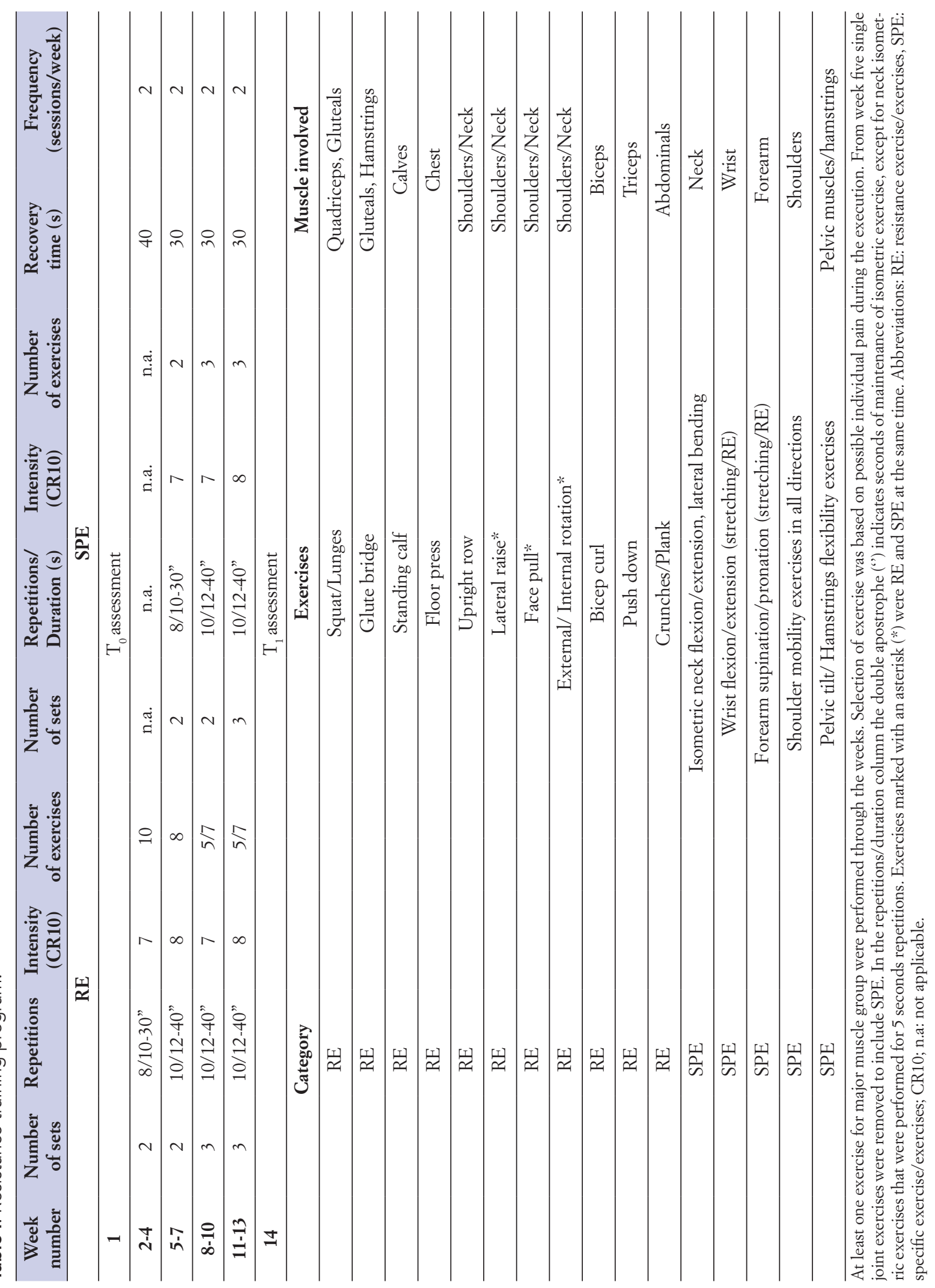

Muscles, Ligaments and Tendons Journal 2021;11 (1) 
Table II. Sociodemographic characteristics of the study participants (mean \pm standard deviation).

\begin{tabular}{ll}
\hline Variables & Participants (18) \\
\hline Sociodemographic characteristics & \\
\hline Age (years) & $49.11(7.58)$ \\
\hline Weight $(\mathrm{Kg})$ & $82.46(14.59)$ \\
\hline Height $(\mathrm{m})$ & $1.74(0.06)$ \\
\hline BMI $\left(\mathrm{Kg} * \mathrm{~m}^{-2}\right)$ & $27.19(4.64)$ \\
\hline Musculoskeletal disease & $4.78(3.08)$ \\
\hline LBP $(\mathrm{L}-\mathrm{VAS})$ & 4 \\
\hline Upper limb disease $(\mathrm{n})$ & 1 \\
\hline Neck disease (n) & 5 \\
\hline Low back disease (n) & 274.72 (139.44) \\
\hline GPAQ & 4 \\
\hline Daily sedentary behaviors (min) & 3 \\
\hline Sedentary participants (n) & 11 \\
\hline Low level of physical activity (n) & \\
\hline Active participants (n) & \\
\hline $\begin{array}{l}\text { M: male, BMI: body mass index; LBP: low back pain; GPAQ: global phys- } \\
\text { ical activity questionnaire. }\end{array}$
\end{tabular}

Results are summarized in table III. The attendance was $83.42 \%(\mathrm{SD}=14.09 \%, \mathrm{IQR}=76.04-92.30$, Minimal value $=70.83 \%)$.

Cardiorespiratory endurance evaluated with 2MST

showed a significant increase of 14.71 steps $(\mathrm{p}=0.01$, $\mathrm{ES}=0.64$.
Flexibility of lower and upper limb increased, but only CSRT recorded significant changes. In details, right and left CSRT showed significant improvement of 4.31 ( $R, p=0.03$, $\mathrm{ES}=0.38)$ and $4.06(\mathrm{~L}, \mathrm{p}=0.04, \mathrm{ES}=0.35)$. Upper limb flexibility improved without statistical significance.

Muscular strength improved in both right and left handgrip isometric strength. The strength of right hand recorded an increase of $3.77 \mathrm{~kg}(\mathrm{p}=0.01, \mathrm{ES}=0.43)$, while left hand recorded and increase of $2.22 \mathrm{~kg}(\mathrm{p}=0.02, \mathrm{ES}=0.3)$.

Pain and disabilities outcomes showed significant reduction of DASH scores $(-4.28 ; \mathrm{p}=0.03$; ES $=0.29)$, while L-VAS recorded a reduction on pain but without statistical significance $(-0.56 ; \mathrm{p}=0.38)$.

\section{DISCUSSION}

The aim of this pilot study was to evaluate the effect of a supervised resistance-training program on physical capacity, pain perception and disability. Moreover, another point of interest was to verify the feasibility of the intervention performed into the company considering individual conditions, the strenuous working activity and the full freedom of choice to take part to the training sessions. The supervised $\mathrm{PE}$ protocols induced the improvement of cardiorespiratory endurance, muscular strength of upper limb and flexibility of lower limb. Moreover, DASH score decreased significantly after intervention $(\mathrm{p}=0.03)$, while L-VAS decreases without statistical significance $(\mathrm{p}=0.38)$.

Low back pain is a multifactorial disease that involve physical and psychological sphere. Even if exercise is one of the most efficient strategies for the management and prevention

Table III. Results of outcome measures.

\begin{tabular}{|c|c|c|c|c|c|c|}
\hline Variables & $\mathrm{T}_{0}$ & $\mathrm{~T}_{1}$ & Difference & P-value & $\begin{array}{c}95 \% \\
\text { Confidence } \\
\text { Intervals (CI) }\end{array}$ & Effect size \\
\hline \multicolumn{7}{|l|}{ Pain and disabilities } \\
\hline L-VAS & $4.78(3.08)$ & $4.22(3.46)$ & -0.56 & 0.38 & $-1.85,0.74$ & 0.18 \\
\hline DASH & $20.69(16.20)$ & $16.04(12.95)$ & -4.28 & 0.03 & $0.06,8.62$ & 0.29 \\
\hline \multicolumn{7}{|c|}{ Health-related physical fitness } \\
\hline 2-minutes step test & $76.29(22.81)$ & $91.00(22.50)$ & 14.71 & 0.01 & $4.24,25.17$ & 0.64 \\
\hline Chair sit and reach $\mathrm{R}(\mathrm{cm})$ & $-9.54(11.31)$ & $-5.22(10.64)$ & -4.31 & 0.03 & $-8.15,-0.48$ & 0.38 \\
\hline Chair sit and reach $\mathrm{L}(\mathrm{cm})$ & $-7.71(11.58)$ & $-3.65(10.02)$ & -4.06 & 0.04 & $-7.90,-0.21$ & 0.35 \\
\hline Back scratch R $(\mathrm{cm})$ & $-0.86(7.16)$ & $0.25(7.67)$ & 1.11 & 0.18 & $-0.54,2.76$ & 0.16 \\
\hline Back scratch L $(\mathrm{cm})$ & $-4.90(8.75)$ & $-3.55(7.98)$ & 1.35 & 0.16 & $-0.60,3.30$ & 0.15 \\
\hline Handgrip test $\mathrm{R}(\mathrm{kg})$ & $42.81(8.76)$ & $46.58(7.87)$ & 3.77 & 0.01 & $1.06,6.48$ & 0.43 \\
\hline Handgrip test $\mathrm{L}(\mathrm{kg})$ & $40.63(7.42)$ & $42.84(5.89)$ & 2.22 & 0.02 & $0.40,4.03$ & 0.30 \\
\hline
\end{tabular}

L-VAS: Visual Analogue Scale score for low back; DASH: Shoulder and Hand Questionnaire score; R: Right; L: Left; PA: physical activity. 
of LBP (19), our results showed contrast results. The analysis of participants showed a reduction of pain in 5 out 18 participants, an unchanged pain in 6 out 18, while 7 participants worsened their LBP. Focusing on worsened participants, the difference with who improved or unchanged is the age, with 5 out 7 that aged more than 50 years old. Our hypothesis is that older workers could be affected by chronic LBP and needs longer and different exercise protocol for the management of pain.

Several studies reported clinical meaningful reduction of neck and shoulder pain and improvement of shoulder range of motion after RT program (20). Moreover, shoulders ROM were associated with severity of pain suggesting the needs of specific intervention for the management of this condition (21). Indeed, our study, RT programs induced the significant improvement of DASH scores, even if the shoulder flexibility improved without statistical significance (right, $\mathrm{p}=0.18$, left, $\mathrm{p}=0.16$ ). Moreover, upper limb grip strength improved significantly for both right $(+8.81 \%, \mathrm{p}$ $=0.01)$ and left $(+5.45 \%, \mathrm{p}=0.02)$ grip strength. Handgrip test seems to be related with musculoskeletal pain and disabilities. The improvement of grip strength could represent an improvement in overall body strength that could be related to decrease in neck, shoulders and hand disability through reduction in relative workload (22).

Chair sit and reach test is an indirect measure of hamstrings flexibility that is a physical capacity often related to LBP (23). Resistance training program induced an improvement of right $(-45.25 \%, \mathrm{p}=0.03)$ and left $(-52.62 \%$, p $=0.04$ ) lower limb flexibility, with a reduction of L-VAS. However, pain reduction was not statistically significant due to the physical exertion demands. In fact, heavy physical work, dynamic working postures and weight lifting are risk factors for low back pain (24), and this type of exercise protocol is not enough for the significant reduction of pain. Commonly, lower level of aerobic capacity is associated with an increased risk of chronic LBP (25). Even if the PE program was composed mainly by strength and flexibility exercises, participants improved their cardiovascular capacity $(+19.28, \mathrm{p}=0.01)$. Probably, the improvement could be related to the general physical conditioning induced by the specific and structured exercise program.

The second aim of this study was the analysis of the attendance and relative feasibility of the intervention in the workplace. Generally, the adherence to health promotion interventions program at workplace were below that $50 \%$ (26). For this reason, we hypothesized that the strenuous working activity and the fear of worsening the previous musculoskeletal pain could act as deterrent to RT program participation immediately after the shift. Instead, the mean adherence was $83.42 \%$ (IQR $=76.04 \%-92.30 \%)$. Probably, the occupational doctor prescription for RT program may have favored the participation.

\section{Limitations}

This study had some limitations. Firstly, group pretestposttest design of this study is the major limitation; in fact, the absence of a control group prevents the attribution of results to the RT program. Moreover, the pilot nature of this study partially justifies this design. In fact, one of our objectives was the evaluation of the feasibility of the intervention for future research implementation. Secondly, the heterogeneity of the sample could be another important limitation, but the real-world setting exposes to heterogeneity itself. Thirdly, pain and disability evaluation could be more complete using VAS in different body regions or the Neck Pain and Disability Scale. Because the evaluation was carried out after the shift, the need of a brief evaluation overcomes the desire of completeness. Fourthly, considering the exercise protocol, another limitation consisted on the lack of data about mean environmental temperature during the session. Finally, we did not perform the intention to treat analysis because two participants drop out, and they did not perform the post treatment evaluation.

\section{CONCLUSIONS}

This study demonstrates the feasibility of a RT intervention in a real work setting after the working shift. Even though participants were engaged in strenuous working activity, spontaneous attendance was $83.42 \%$. Moreover, RT seems to be effective in reducing pain perception and disability related to WRMSDs, particularly in the joints involved in the exercises. Hence, specific exercises should be added to RT program in the exercise prescription to reach better results, considering specific risk factors and joints involve in working activity.

\section{CONTRIBUTIONS}

S.G., M.B. designed the work; B.V., F.D. acquired the data; V.B., E.R. analyze the data; L.C., A.D.B, S.M. provide their intellectual contribution about data interpretation; D.S.B., C.L.A., G.P., D.C., A.E., M.B. participate in manuscript writing, give an important contribution in revising it and the final draft. All authors give their final approval of the version to be published.

\section{CONFLICT OF INTERESTS}

The authors declare that they have no conflict of interests. 


\section{REFERENCES}

1. Aptel M, Aublet-Cuvelier A, Cnockaert JC. Work-related musculoskeletal disorders of the upper limb. Joint Bone Spine 2002;69(6):546-55.

2. Forde MS, Punnett L, Wegman DH. Pathomechanisms of work-related musculoskeletal disorders: conceptual issues. Ergonomics 2002;45(9):619-30.

3. Rasotto C, Bergamin M, Simonetti A, et al. Tailored exercise program reduces symptoms of upper limb work-related musculoskeletal disorders in a group of metalworkers: A randomized controlled trial. Man Ther 2015;20(1):56-62.

4. Baldwin ML. Reducing the costs of work-related musculoskeletal disorders: targeting strategies to chronic disability cases. J Electromyogr Kinesiol 2004;14(1):33-41.

5. Gobbo S, Bullo V, Bergamo M, et al. Physical exercise is confirmed to reduce low back pain in office workers: a systematic review of the evidence to imporve best practices in the workplace. J Funct Morphol Kinesiol 2019;4(43).

6. Sihawong R, Janwantanakul P, Sitthipornvorakul E, Pensri P. Exercise therapy for office workers with nonspecific neck pain: a systematic review. J Manipulative Physiol Ther 2011;34(1):62-71.

7. Hagg GM. Human muscle fibre abnormalities related to occupational load. Eur J Appl Physiol 2000;83(2-3):159-65.

8. Coury H, Moreira R, Dias NB. Evaluation of the effectiveness of workplace exercise in controlling neck, shoulder and low back pain: a systematic review. Brazil J Phys Ther 2009;13(6).

9. Alzahrani H, Mackey M, Stamatakis E, Zadro JR, Shirley D. The association between physical activity and low back pain: a systematic review and meta-analysis of observational studies. Sci Rep 2019;9(1):8244.

10. Rikli RE, Jones CJ. Development and validation of a functional fitness test for community-residing older adults. J Aging Phys Act. 1999;7(2):129-61.

11. Roberts HC, Denison HJ, Martin HJ, et al. A review of the measurement of grip strength in clinical and epidemiological studies: towards a standardised approach. Age Ageing 2011;40(4):423-9.

12. Padua R, Padua L, Ceccarelli E, et al. Italian version of the Disability of the Arm, Shoulder and Hand (DASH) questionnaire. Cross-cultural adaptation and validation. J Hand Surg Br 2003;28(2):179-86.

13. Hawker GA, Mian S, Kendzerska T, French M. Measures of adult pain: Visual Analog Scale for Pain (VAS Pain), Numeric Rating Scale for Pain (NRS Pain), McGill Pain Questionnaire (MPQ), Short-Form McGill Pain Questionnaire (SF-MPQ), Chronic Pain Grade Scale (CPGS), Short Form-36 Bodily Pain Scale (SF-36 BPS), and Measure of Intermittent and Constant
Osteoarthritis Pain (ICOAP). Arthritis Care Res (Hoboken) 2011;63 Suppl 11:S240-52.

14. Organization WH. Global Physical Activity Questionnaire (GPAQ): Analysis Guide Available at: https://www.who.int/ ncds/surveillance/steps/resources/GPAQ_Analysis_Guide. pdf. Last access date: 17/01/2020.

15. Buckley JP, Borg GA. Borg's scales in strength training; from theory to practice in young and older adults. Appl Physiol Nutr Metab 2011;36(5):682-92.

16. Team RC. R: a language and environment for statistical computing. Vienna, Austria: R Foundation for statistical Computing; 2018.

17. Cohen J. Statistical Power Analysis for the Behavioral Sciences: Taylor \& Francis; 2013.

18. Padulo J, Oliva F, Frizziero A, Maffulli N. Basic principles and recommendations in clinical and field Science Research: 2018 update. MLTJ 2018;8(3):305-7.

19. Huang R, Ning J, Chuter VH, et al. Exercise alone and exercise combined with education both prevent episodes of low back pain and related absenteeism: systematic review and network meta-analysis of randomised controlled trials (RCTs) aimed at preventing back pain. Br J Sports Med. 2019.

20. Ylinen J, Takala EP, Nykanen M, et al. Active neck muscle training in the treatment of chronic neck pain in women: a randomized controlled trial. JAMA. 2003;289(19):2509-16.

21. Anwer S, Alghadir AH, Al-Eisa ES, Iqbal ZA. The relationships between shoulder pain, range of motion, and disability in patients with shoulder dysfunction. J Back Musculoskelet Rehabil 2018;31(1):163-7.

22. Coury H, Moreira R, Dias NB. Evaluation of the effectiveness of workplace exercise in controlling neck, shoulder and low back pain: a systematic review. Revista Brasileira de Fisioterapia 2009;13(6):461-79

23. Sadler SG, Spink MJ, Ho A, De Jonge XJ, Chuter VH. Restriction in lateral bending range of motion, lumbar lordosis, and hamstring flexibility predicts the development of low back pain: a systematic review of prospective cohort studies. BMC Musculoskelet Disord. 2017;18(1):179.

24. da Costa BR, Vieira ER. Risk factors for work-related musculoskeletal disorders: A systematic review of recent longitudinal studies. Am J Ind Med 2010;53(3):285-323.

25. Wormgoor ME, Indahl A, van Tulder MW, Kemper HC. The impact of aerobic fitness on functioning in chronic back pain. Eur Spine J. 2008;17(4):475-83.

26. Robroek SJ, van Lenthe FJ, van Empelen P, Burdorf A. Determinants of participation in worksite health promotion programmes: a systematic review. Int J Behav Nutr Phys Act 2009;6:26. 